\title{
TLRs find a partner in crime
}

A recent study published in Nature Immunology describes a new mechanism of sterile inflammation that is common to atherosclerosis and Alzheimer's disease. It shows that the recognition of altered self components that aggregate in plaques in these two diseases by the scavenger receptor CD36 triggers the assembly of Toll-like receptor 4 (TLR4) and TLR6 heterodimers, leading to the induction of pro-inflammatory responses that underlie the pathology of these diseases.

The deposition of oxidized low density lipoprotein (oxLDL) and amyloid- $\beta$ peptide in plaques in atherosclerosis and Alzheimer's disease, respectively, is known to contribute to the inflammatory events associated with these diseases.

Although the inflammation is sterile, the TLR signalling adaptor myeloid differentiation primary-response protein 88 (MYD88) is required. By carrying out a series of experiments with macrophages lacking various TLRs, the authors found that TLR4 and TLR6, but not TLR2, were required for the induction of chemokines and interleukin- $1 \beta$ (IL-1 $\beta$ ) in response to oxLDL.
Moreover, TLR4 and TLR6 seemed to function cooperatively, as cells expressing both TLRs showed a sixfold increase in nuclear factor- $\kappa \mathrm{B}$ $(\mathrm{NF}-\kappa \mathrm{B})$ activation following exposure to oxLDL compared with cells expressing the receptors individually. This suggests the existence of a previously undescribed heterodimer of TLR4 and TLR6.

To understand how oxLDL triggers TLR signalling, the authors assessed the role of CD14 (a TLR4 co-receptor required for lipopolysaccharide recognition) and CD36 (a lipid-binding scavenger receptor previously shown to function as a TLR2 co-receptor). Whereas CD14 did not increase oxLDL-induced NF- $\kappa B$ activation, CD36 led to a threefold increase in NF- $\kappa \mathrm{B}$ activation in cells expressing both TLR4 and TLR6. A role for CD36 in the inflammatory response was also shown in vivo: atherosclerosis-prone $\left(\right.$ Apoe $\left.^{-/-}\right)$mice that lack CD36 expression showed a lower production of chemokines in the descending aorta and developed fewer atherosclerotic lesions than CD36-sufficient $A p o e^{-/-}$ mice when fed a high-fat diet.

The collaboration between CD36, TLR4 and TLR6 was also found to be involved in inflammation induced by Alzheimer's diseaseassociated amyloid- $\beta$. Amyloid- $\beta$ peptide induced NF- $\kappa \mathrm{B}$ activation in a reporter cell line expressing TLR4 and TLR6, and this was further enhanced when CD36 was overexpressed. In microglial cells, amyloid- $\beta$ peptide induced the secretion of nitric oxide, reactive oxygen species and IL- $1 \beta$, all of which have been implicated in the cytotoxic effects of amyloid- $\beta$ on neurons. Accordingly, amyloid- $\beta$ peptide led to the widespread death of neuronal cells when cultured with wild-type microglial cells but not with $T l r 4^{-/-}$or $\operatorname{Tlr}^{-/-}$microglial cells. Together, these findings suggest that a macromolecular signalling complex of CD36, TLR4 and TLR6 mediates a pathogenic innate immune response to endogenous CD36 ligands.

Finally, immunoprecipitation and microscopy studies revealed that the presence of oxLDL triggers co-localization of CD36, TLR4 and TLR6 mainly in intracellular compartments, and that this interaction and downstream signalling depend on tyrosine residue 463 in the cytoplasmic carboxyl terminus of CD36. Further experiments indicated that association of the kinase LYN with CD36 Tyr463 initiates TLR4-TLR6 heterodimerization and signalling.

So, this study shows that by partnering with a scavenger receptor, TLRs can be triggered in response to endogenous proteins and induce innate immune responses underlying sterile inflammatory diseases.

Lucy Bird

ORIGINAL RESEARCH PAPER Stewart, C. R. et al. CD36 ligands promote sterile inflammation through assembly of a Toll-like receptor 4 and 6 heterodimer. Nature Immunol. 27 Dec 2009 (doi:10.1038/ni1836) 